\title{
COMMUNICATION
}

\section{Emulsion-templated silica nanocapsules formed using bio-inspired silicification $\dagger$}

Cite this: DOI: $10.1039 / \times 0 \times x 00000 x$

\author{
D. Wibowo, ${ }^{a}$ C.-X. Zhao ${ }^{a}$ and Anton P. J. Middelberg*a
}

Received 00th January 2014,

Accepted 00th January 2014

DOI: $10.1039 / \times 0 \times x 00000 x$

www.rsc.org/chemcomm

A novel, bio-inspired templating platform technology is reported for synthesis of biocompatible oil-core silica-shell nanocapsules with tunable shell thickness by utilizing a designed bifunctional peptide. Furthermore, facile encapsulation of an active molecule and its sustained release are demonstrated.

Templated core-shell silica nanocapsule structures promise great impact from the intersection of molecular and inorganic chemistry. ${ }^{1}$ They allow encapsulation of fluorescent materials, ${ }^{2}$ magnetic nanoparticles, ${ }^{3}$ and active agents ${ }^{4}$ in the core domain for imaging, sensing, and delivery, with a higher loading-capacity than an equivalent solid nanoparticle. The silica shell is engineered around the core to provide: 1) a protective envelope with chemical and mechanical stability for storage and delivery; 2) accessible pathways for adsorption, separation and sustained release; and 3) ease of surface modification with optical, magnetic, and/or biological functionalities enhancing the performance of nanocapsules for applications including biolabelling, controlled release and targeted delivery. Therefore, the fabrication of silica nanocapsules has attracted much research and industrial interest.

Hard- and soft-templating approaches are commonly employed to construct silica nanocapsules. Soft-templating methods offer a number of advantages. Thermal decomposition or chemical dissolution necessary for complete removal of the solid core is avoided, and the loading of an active cargo is simplified as it can be solubilized in the core prior to silica shell formation. ${ }^{5}$ Sol-gel routes, ${ }^{5-6}$ including self-templating of an alkoxysilane ${ }^{5 b}$, 6a and interfacial poly-condensation of hydrolyzed silicon alkoxide on ionic $^{5 \mathrm{a}}$ and non-ionic surfactants, ${ }^{6 \mathrm{~b}}$ have been developed to synthesize silica shells on oil-in-water $(\mathrm{O} / \mathrm{W})$ emulsions in the nanometer range. However, these soft-templating approaches variously incorporate the use of elevated temperature or extreme $\mathrm{pH}$, or involve chemicals that have limited biocompatibility. An alternative pathway using more benign reaction conditions and biocompatible components would, for some applications, remove limitations embedded in current approaches.

Biomimetic templating offers mild routes to silica nanostructures using biomolecules. ${ }^{7}$ Identification of amino acid sequences from nature $^{8}$ and combinatorial biology ${ }^{9}$ capable of catalyzing silica formation has led to new strategies. ${ }^{10}$ These involve biosilicification of the biomolecules in bulk aqueous solution ${ }^{10 \mathrm{a}-\mathrm{c}}$ and at solid-fluid interfaces. ${ }^{10 \mathrm{~d}}$ Erni et al. reported the synthesis of silica capsules at a scale of hundreds of micrometers by silicification of cross-linked coacervate of gelatin and Acacia gum around oil droplets. ${ }^{11}$ To the best of our knowledge, peptides or proteins with modularized amino acid sequences capable of both stabilizing an emulsion and inducing biosilicification at the oil-water interface have not been reported, especially for the fabrication of silica capsules of nanometer dimension. Here, we designed a bifunctional peptide comprising one module capable of forming a stable nanoemulsion core and another module that induces biosilicification at oil-water interface to facilitate formation of oil-filled silica nanocapsule. This work opens new environmentally-friendly strategies for bioinspired oil-core silica nanocapsule formation having wide potential utility.

We designed a bifunctional peptide (SurSi, AcMKQLAHSVSRLEHA RKKRKKRKKRKKGGGY-CONH 2 ) by modularizing part of peptide surfactant AM1 (AcMKQLADSLHQLARQVSRLEHA-CONH 2 ) for surface activity ${ }^{12}$ (i.e., Sur module, MKQLAHSVSRLEHA) with a sequence for biosilicification (i.e., $\mathrm{Si}$ module, RKKRKKRKKRKKGGGY). Peptide surfactant AM1 is capable of reversibly and precisely controlling the stability of $\mathrm{O} / \mathrm{W}$ emulsions ${ }^{12}$ by forming a cohesive interfacial film responsive to metal ion binding to histidine $(\mathrm{H})$ residues. ${ }^{13}$ Sur was designed based on heptads of AM1 but without the second heptad (i.e., LHQLARQ) and with the replacement of aspartic acid (D) with histidine (H) in the first heptad. It is known that the molecular weight of adsorbing peptide molecules at oilwater interfaces $(M)$ is inversely proportional to the square of the interfacial adsorption rate constant $\left(k_{\mathrm{a}}\right) .{ }^{14}$ Therefore, the incorporation of only two heptads, instead of three heptads, of AM1 in Sur would promote more rapid adsorption of the peptide at a freshly formed oil-water interface. Rapid interfacial adsorption facilitates efficient coverage of peptide molecules onto oil droplets and hence formation and stabilization of nanoscale emulsions. ${ }^{15}$ The replacement of aspartic acid (D) with histidine $(\mathrm{H})$ was designed to have the same number of histidine residues as AM1, thus allowing the metal-ion-mediated crosslinking between interfacially adsorbed peptide molecules and the formation of a cohesive film state at the interface $^{16}$ (Scheme 1). Proteins and peptides having positivelycharged amino acids arginine $(\mathrm{R})$ and lysine $(\mathrm{K})$ can induce formation of silica under mild conditions. ${ }^{8 b, 9 a, 10 a}$ The R- and K-rich 
Si module, based on a sequence known to precipitate titania in bulk, ${ }^{17}$ is discovered here to promote biosilicification at the oilwater interface when modularized with Sur. A number of peptide- or protein-based biomineralization mechanisms have been proposed in literature, including nucleophilic attack, hydrogen bonding, and electrostatic attraction or ion-pairing. Cha et al. reported that polar amino acid residues, including serine $(\mathrm{S})$, tyrosine $(\mathrm{Y})$, histidine $(\mathrm{H})$ and glutamine $(\mathrm{Q})$, can promote nucleophilic attack, in which the nucleophilicity of hydroxyl oxygen of serine can be enhanced by the formation of hydrogen bond between serine and adjacent histidine, on the silicon atom within tetraethoxysilane ${ }^{8 a}$, 10a (TEOS) and thus may facilitate its hydrolysis to produce silanol and negativelycharged silanolate (Scheme 1). Moreover, positively-charged arginine (R) and lysine (K) may interact electrostatically with silanolate at near-neutral $\mathrm{pH}$, while the hydroxyls of serine $(\mathrm{S})$ and tyrosine (Y) promote hydrogen bonding with silanol ${ }^{8 \mathrm{~b}}$ (Scheme 1), which in turn act as nucleation sites and direct silica growth through poly-condensation of the silica species at the interface. Under these possible mechanisms, SurSi peptide would thus be expected to make silica nanocapsules at room temperature and near-neutral $\mathrm{pH}$ without using any chemical-based catalysts.

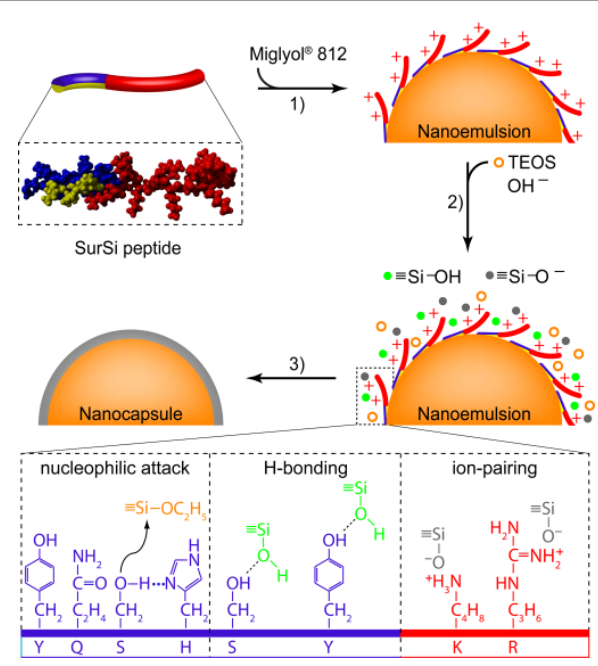

Scheme 1 Synthesis strategy of silica nanocapsule based on bio-inspired silicification at an oil-water interface. Step 1) sonication of Miglyol $^{\circledR} 812$ oil in a SurSi solution followed by dialysis at $\mathrm{pH}$ 7.5; step 2) addition of tetraethoxysilane (TEOS) to nanoemulsion; and step 3) interfacial poly-condensation of silica species. SurSi peptide operates synergistically by 1 ) stabilizing a nanoemulsion core through a "Sur" module incorporating hydrophobic amino acids (in yellow) to impart facial amphiphatic character hence promoting adsorption at the nanoemulsion interface; and 2) directing nucleation and growth of silica at the oil-water interface through a "Si" module.

Nanoemulsion was formed by sonication of Miglyol ${ }^{\circledR} 812$ oil in SurSi solution, confirming surface activity due to the amphiphilic Sur module. The addition of $32 \mu \mathrm{mol}$ TEOS to dialyzed nanoemulsion at pH 7.5 or 8 led to successful formation of silica nanocapsule after $20 \mathrm{~h}$, as evidenced by an electron-dense shell (Fig. 1a,b). The outer diameter and the shell thickness of nanocapsules formed at $\mathrm{pH} 7.5$ as measured by transmission electron microscopy (TEM) were $66 \pm 5 \mathrm{~nm}$ and $12 \pm 2 \mathrm{~nm}$, respectively (Fig. 1a). Dynamic light scattering (DLS) confirmed a size increase in comparison with base nanoemulsion (Fig. S1a). Additionally, the $\zeta$-potential of both the nanoemulsion templates and the nanocapsules were measured. In contrast to the nanoemulsions which had a positive zeta potential of $+35 \pm 5 \mathrm{mV}$ because of the positivelycharged SurSi peptide, the silica nanocapsules exhibited a highly negative charge of $-30 \pm 5 \mathrm{mV}$ as a result of silanolate groups on the silica surface. Furthermore, formation of a silica shell was confirmed by presence of silicon and oxygen in the thick layer surrounding the oil core using energy-dispersive $\mathrm{X}$ ray spectroscopy (EDX) (Fig. 1). As a control experiment, AM1 was unable to demonstrate an effective dual functionality of emulsion stabilization and silica shell formation, as the silica was largely formed in bulk solutions rather than at oil-water interfaces (Fig. S2). Thus modularization of a partial AM1 sequence with a specialized $\mathrm{Si}$ sequence was considered essential for acceptable practical formation of nanocapsules. The results confirm this is the case, with the desired result achieved in a way not possible with AM1.

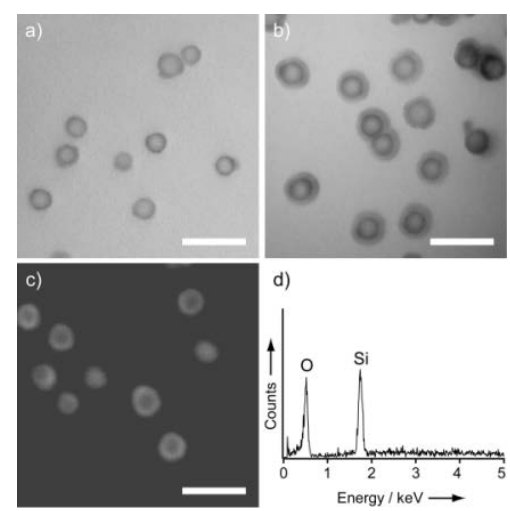

Fig. 1 Silica nanocapsules formed using bio-inspired silicification. (a, b) Transmission electron microscopy (TEM) images confirming the formation of a nanostructured core-shell architecture after a 20-h biosilicification reaction of SurSi-stabilized nanoemulsion with $32 \mu \mathrm{mol}$ TEOS in $25 \mathrm{~mm}$ HEPES buffer at $\mathrm{pH}$ 7.5 (a) or at $\mathrm{pH} 8$ (b). (c, d) Representative TEM dark-field image (c) and the corresponding energy-dispersive X-ray spectrum (d) of the nanocapsule confirming existence of a silica shell. Scale bars are $200 \mathrm{~nm}$.

The silica shell thickness is tunable. Compared to reaction at $\mathrm{pH}$ 7.5 , reaction at $\mathrm{pH} 8$ with $32 \mu \mathrm{mol}$ TEOS for $20 \mathrm{~h}$ gave a remarkable increase in silica shell thickness (27 $\pm 6 \mathrm{~nm}$ ) (Fig. 1b). DLS also confirmed a size increase with increasing reaction $\mathrm{pH}$ (Fig. S1b). As increasing reaction $\mathrm{pH}$ assists TEOS hydrolysis and generates a higher concentration of reactive silica species, we hypothesized that increasing the concentration of negatively-charged silanolate-rich species would favor their attraction to the positively-charged nanoemulsion surface. Then the positively-charged amino acids enriched at the oil-water interface would generate highly localized electrostatic forces to the silica species. Another silica species, silanol, could also interact with hydrogen-bond donor amino acids at the interface through hydrogen-bonding (Scheme 1). The attracted silica species then could act as nucleation sites for further silica growth forming the shell with thickness solely depending on the local concentration of the silica species.

To further investigate silica formation and methods for control of silica shell thickness, reaction time and TEOS concentration were varied at pH 7.5 (Fig. 2a). Shell thicknesses increased regularly with increasing reaction time and TEOS concentration, as reflected by an increase in nanocapsule diameter (Fig. 2b) shown in TEM. The boundary between core and shell by TEM was more obvious after 30-h reaction (Fig. 2a), and the silica shell thicknesses, as measured by TEM, were $5 \pm 1 \mathrm{~nm}, 10 \pm 2 \mathrm{~nm}$ and $16 \pm 3 \mathrm{~nm}$ following reaction with $8 \mu \mathrm{mol}, 16 \mu \mathrm{mol}$ and $32 \mu \mathrm{mol}$ TEOS, respectively. The surface of the silica shell was rough, especially during the first $20 \mathrm{~h}$, as observed from TEM images (Fig. 2a). This roughness might be due to the fact that the silica species were still undergoing polycondensation at the interface as the reaction proceeded. ${ }^{18}$ The silica 
shell kept on growing through further interfacial biosilicification and poly-condensation, and became thicker over time until TEOS was depleted (Fig. 2b). The evolution of the silica growth during biosilicification reaction was also monitored using DLS. An increase of the nanocapsule diameter was observed at different reaction times (Fig. S3). These results demonstrate the possibility of obtaining well-dispersed nanocapsules (see PDIs in Table S1) having different silica shell thicknesses by controlling TEOS concentration and reaction time.

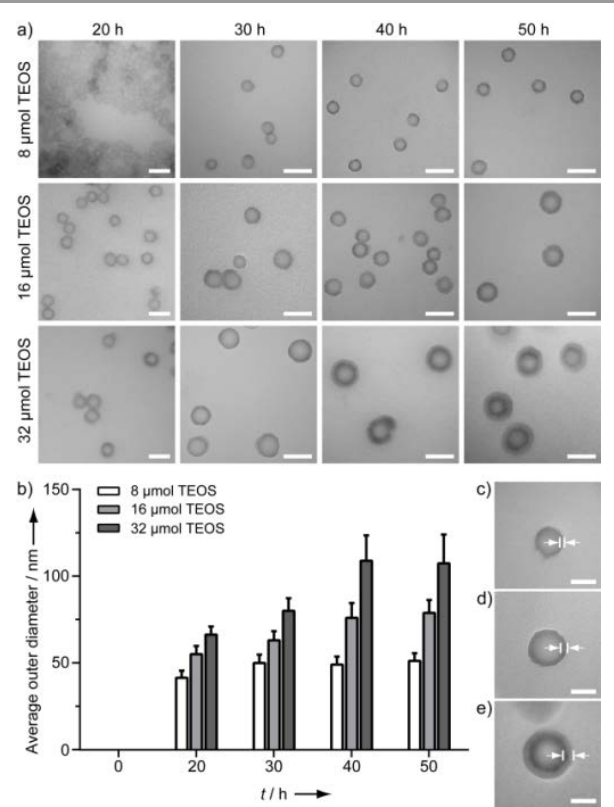

Fig. 2 Tunable silica shell thickness through control of biosilicification reaction time $(t)$ and TEOS concentration. (a) TEM images showing silica nanocapsules produced at different reaction times and TEOS concentrations at $\mathrm{pH} 7.5$ (scale bars are $100 \mathrm{~nm}$ ). (b) Increases in the average outer diameter of nanocapsules reflected increases in silica shell thicknesses as calculated from TEM based on the average value of at least 100 individual nanocapsules. (c-e) Representative TEM images of individual nanocapsules after a 50-h biosilicification reaction with $8 \mu \mathrm{mol}$ (c), $16 \mu \mathrm{mol}$ (d) and $32 \mu \mathrm{mol}$ (e) TEOS (scale bars are $50 \mathrm{~nm}$ ).

Silica nanocapsules can be used to encapsulate an active material in the oil core for protection and/or to provide a diffusion barrier through the silica shell for slow release of the active, ${ }^{5}$ thus enabling applications in biomedical and agricultural domains. We demonstrate facile encapsulation and sustained release profile associated with the core-shell structure of a silica nanocapsule using fipronil as a model of oil-soluble active molecules. Fipronil is a phenylpyrazole insecticide that controls a broad spectrum of insect pests and provides long-term protection for crops. ${ }^{19}$ Fipronil can be solubilized in Miglyol ${ }^{\circledR} 812$ oil at high loading capacity (up to $10 \mathrm{mg}$ $\mathrm{mL}^{-1}$ ). We loaded $10 \mathrm{mg} \mathrm{mL}^{-1}$ fipronil in oil to form a SurSistabilized nanoemulsion. After a 30-h reaction with TEOS at $\mathrm{pH} 7.5$, TEM images (Fig. 3a-c) suggested the successful formation of silica nanocapsules. The encapsulation efficiencies of fipronil in the silica nanocapsules obtained at TEOS concentrations of $16 \mu \mathrm{mol}, 32 \mu \mathrm{mol}$ and $96 \mu \mathrm{mol}$ were $69.3 \%, 70.6 \%$ and $71.1 \%$, respectively (see ESI $\dagger$ ). With a fipronil-loaded nanoemulsion, the silica shell thickness can also be controlled by varying TEOS concentration (Fig. 3a). Increase in TEOS concentration $(16 \mu \mathrm{mol}, 32 \mu \mathrm{mol}$ and 96 $\mu \mathrm{mol})$ resulted in loaded nanocapsules with higher average diameters (140 $\pm 13 \mathrm{~nm}, 198 \pm 15 \mathrm{~nm}$ and $288 \pm 24 \mathrm{~nm}$, respectively) and thicker silica shells $(8 \pm 2 \mathrm{~nm}, 25 \pm 3 \mathrm{~nm}$ and $44 \pm 7 \mathrm{~nm}$, respectively) (Fig. 3a).
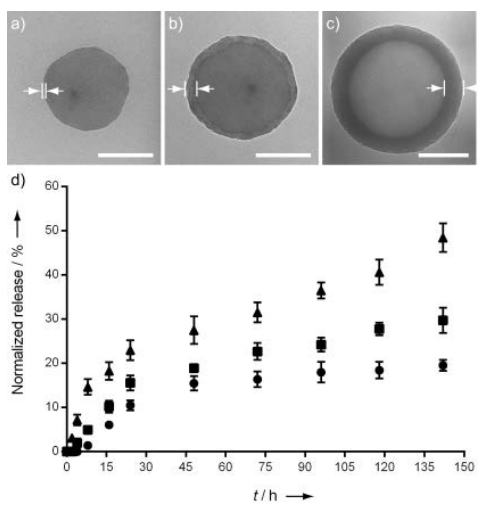

Fig. 3 Encapsulation of fipronil in silica nanocapsules and its sustained release profile. $(a-c)$ TEM images showing fipronil-loaded silica nanocapsules with 8-nm (a), 25-nm (b) and 44-nm (c) shell thicknesses (scale bars are $100 \mathrm{~nm}$ ). (d) Release profiles of fipronil-encapsulated nanocapsules with 8-nm ( $\boldsymbol{\Delta}), 25-\mathrm{nm}$ (घ) and 44-nm (•) silica shell thicknesses.

We further investigated the sustained release of fipronil from silica nanocapsules in water. Fig. 3 shows the fipronil release profiles (Fig. 3b) for silica nanocapsules with shell thickness of $8 \mathrm{~nm}, 25 \mathrm{~nm}$ and $44 \mathrm{~nm}$ (Fig. 3a). The concentration of fipronil in solution resulting from release from the silica nanocapsules to water was normalized against the saturated concentration of fipronil in water $\left(2 \mu \mathrm{g} \mathrm{mL}^{-1}\right)$ to allow normalized comparison of different nanocapsule designs. We observed the effect of silica shell thickness on the release profile of fipronil. Silica nanocapsules with $8 \mathrm{~nm}$ shell thickness showed $3.1 \pm 0.6 \%$ release of fipronil at $2 \mathrm{~h}$. Nanocapsules with a thicker shell, i.e. $25 \mathrm{~nm}$ and $44 \mathrm{~nm}$, demonstrated slower fipronil release which occurred at $4 \mathrm{~h}$ and $8 \mathrm{~h}$ at $1.9 \pm 1.0 \%$ and $1.4 \pm 0.2 \%$, respectively. The fipronil release then gradually increased with time. The release of fipronil decreased with increasing silica shell thickness, with $48.4 \pm 3.2 \%, 29.8 \pm 2.9 \%$ and $19.6 \pm 1.2 \%$ of fipronil released from silica nanocapsules having $8 \mathrm{~nm}, 25 \mathrm{~nm}$ and $44 \mathrm{~nm}$ shell thicknesses, respectively, at 142 h. TEM images (Fig. S4) showed that the shell morphology of the nanocapsules was still intact before and after $142 \mathrm{~h}$ of study, indicating that the release of fipronil from silica nanocapsules in water was governed mainly by diffusion rather than by physical changes to the silica nanoshell. This study demonstrated that the core-shell structure of silica nanocapsules formed using bio-inspired silicification can be used for encapsulation of active components and thus their sustained release, and the release profile can be controlled by the silica shell thickness.

To conclude, we demonstrated a novel and facile method for the fabrication of silica nanocapsules using emulsion templating based on bio-inspired silicification. Our approach was based on the novel design of a bifunctional peptide by modularizing a surface-active peptide sequence with a sequence having biosilicification activity, thus achieving the formation of stable nanoemulsions followed by the nucleation and growth of a defined and controllable silica shell at the oil-water interface under benign conditions, including room temperature, neutral $\mathrm{pH}$ and without use of toxic reagents. Moreover, the silica shell thickness could be tuned simply by controlling $\mathrm{pH}$, reaction time and silica precursor concentration. Active ingredient was encapsulated effectively in the oil core of silica nanocapsules with a high loading capacity, and was shown to have a sustained release profile dependent on shell thickness. Therefore, the emulsion and biomimetic dual-templating technology developed in this work represents a new strategy for 
forming nanomaterials having core-shell structure using components and processes expected to have minimal environment footprint, opening opportunities for further applications that demand biocompatibility, facile manufacture and high loading capacity coupled with slow release of an active agent.

This work was supported by the Australian Research Council (ARC) (grants DP1093056 and 120103683). DW acknowledges a scholarship from The University of Queensland (UQ). CXZ acknowledges financial support from ARC (Australian Postdoctoral Fellow DP110100394). APJM acknowledges support from the Queensland Government through award of the 2010 Queensland Premier's Science Fellowship. We thank Lei Yu for support with EDX analyses as well as the facilities, and the scientific and technical assistance of the Australian Microscopy \& Microanalysis Research Facility at the Centre for Microscopy and Microanalysis, UQ.

\section{Notes and references}

a Australian Institute for Bioengineering and Nanotechnology, The University of Queensland, Brisbane, QLD 4072 (Australia). E-mail: a.middelberg@uq.edu.au.

$\dagger$ Electronic Supplementary Information (ESI) available: Experimental details and additional figures. See DOI: 10.1039/c000000x/

1. (a) X. W. Lou, L. A. Archer and Z. C. Yang, Adv. Mater., 2008, 20, 3987-4019; (b) A. Guerrero-Martínez, J. Pérez-Juste and L. M. LizMarzán, Adv. Mater., 2010, 22, 1182-1195; (c) W. Schärtl, Nanoscale, 2010, 2, 829-843.

2. A. Burns, H. Ow and U. Wiesner, Chem. Soc. Rev., 2006, 35, 1028-1042.

3. (a) A.-H. Lu, E. L. Salabas and F. Schüth, Angew. Chem. Int. Ed., 2007, 46, 1222-1244; (b) Y. Chen, C. Chu, Y. Zhou, Y. Ru, H. Chen, F. Chen, Q. He, Y. Zhang, L. Zhang and J. Shi, Small, 2011, 7, 2935-2944.

4. (a) J. F. Chen, H. M. Ding, J. X. Wang and L. Shao, Biomaterials, 2004, 25, 723-727; (b) Y. Zhao, L.-N. Lin, Y. Lu, S.-F. Chen, L. Dong and S.H. Yu, Adv. Mater., 2010, 22, 5255-5259.

5. (a) H. Chen, J. He, H. Tang and C. Yan, Chem. Mater., 2008, 20, 58945900; (b) K. Hayashi, M. Nakamura and K. Ishimura, Chem. Commun., 2011, 47, 1518-1520.

6. (a) C. I. Zoldesi and A. Imhof, Adv. Mater., 2005, 17, 924-928; (b) R. S. Underhill, A. V. Jovanovic, S. R. Carino, M. Varshney, D. O. Shah, D. M. Dennis, T. E. Morey and R. S. Duran, Chem. Mater., 2002, 14, 49194925.

7. (a) M. B. Dickerson, K. H. Sandhage and R. R. Naik, Chem. Rev., 2008, 108, 4935-4978; (b) C.-L. Chen and N. L. Rosi, Angew. Chem. Int. Ed., 2010, 49, 1924-1942; (c) S. V. Patwardhan, Chem. Commun., 2011, 47, 7567-7582; (d) S. V. Patwardhan, S. J. Clarson and C. C. Perry, Chem. Commun., 2005, 1113-1121.

8. (a) J. N. Cha, K. Shimizu, Y. Zhou, S. C. Christiansen, B. F. Chmelka, G. D. Stucky and D. E. Morse, Proc. Natl. Acad. Sci. U. S. A., 1999, 96, 361-365; (b) N. Kröger, R. Deutzmann and M. Sumper, Science, 1999, 286, $1129-1132$.

9. (a) R. R. Naik, L. L. Brott, S. J. Clarson and M. O. Stone, J. Nanosci. Nanotechnol., 2002, 2, 95-100; (b) M. Sarikaya, C. Tamerler, A. K. Y. Jen, K. Schulten and F. Baneyx, Nature Mater., 2003, 2, 577-585.

10. (a) J. N. Cha, G. D. Stucky, D. E. Morse and T. J. Deming, Nature, 2000, 403, 289-292; (b) H. R. Luckarift, J. C. Spain, R. R. Naik and M. O. Stone, Nat. Biotechnol., 2004, 22, 211-213; (c) R. R. Naik, M. M. Tomczak, H. R. Luckarift, J. C. Spain and M. O. Stone, Chem. Commun., 2004, 1684-1685; (d) P. Graf, A. Mantion, A. Haase, A. F. Thünemann, A. Maŝić, W. Meier, A. Luch and A. Taubert, ACS Nano, 2011, 5, 820-833.

11. P. Erni, G. Dardelle, M. Sillick, K. Wong, P. Beaussoubre and W. Fieber, Angew. Chem. Int. Ed., 2013, 52, 10334-10338.

12. A. F. Dexter, A. S. Malcolm and A. P. J. Middelberg, Nature Mater., 2006, 5, 502-506.

13. A. F. Dexter and A. P. J. Middelberg, J. Phys. Chem. C, 2007, 111, 10484-10492.
14. A. P. J. Middelberg, C. J. Radke and H. W. Blanch, Proc. Natl. Acad. Sci. U. S. A., 2000, 97, 5054-5059.

15. Y. P. Chuan, B. Y. Zeng, B. O'Sullivan, R. Thomas and A. P. J. Middelberg, J. Colloid Interface Sci., 2012, 368, 616-624.

16. A. P. J. Middelberg, L. He, A. F. Dexter, H. H. Shen, S. A. Holt and R. K. Thomas, J. R. Soc. Interface, 2008, 5, 47-54.

17. M. B. Dickerson, S. E. Jones, Y. Cai, G. Ahmad, R. R. Naik, N. Kröger and K. H. Sandhage, Chem. Mater., 2008, 20, 1578-1584.

18. (a) L. M. Liz-Marzán, M. Giersig and P. Mulvaney, Langmuir, 1996, 12, 4329-4335; (b) Y. Lu, J. McLellan and Y. N. Xia, Langmuir, 2004, 20, 3464-3470.

19. (a) F. Colliot, K. A. Kukorowski, D. W. Hawkins and D. A. Roberts, Proc. Brighton Crop Prot. Conf. Pest Dis. (Brighton, UK), 1992; (b) C. C. D. Tingle, J. A. Rother, C. F. Dewhurst, S. Lauer and W. J. King, Rev. Environ. Contam. Toxicol., 2003, 176, 1-66. 